\title{
Minimal generating orbit sets of torsion elements in $G L_{n}(\mathbb{Z})^{*}$
}

\author{
Jianchun $\mathrm{Wu}^{\dagger}$ (D), Yonghu Zheng \\ Department of Mathematics, Soochow University, Suzhou 215006, CHINA
}

Received 11 May 2020, accepted 11 August 2020, published online 15 March 2021

\begin{abstract}
We construct minimal generating orbit sets for torsion elements in $G L_{n}(\mathbb{Z})$ for $n \leq 4$.
\end{abstract} Keywords: generating orbit sets, rank, torsion, generalized linear group.

Math. Subj. Class.: 15B36, 11C20

\section{Introduction}

Let $\mathbb{Z}^{n}=\mathbb{Z} \times \cdots \times \mathbb{Z}$ be a direct product of $n$ copies of the ring of integers $\mathbb{Z}$. We consider $\mathbb{Z}^{n}$ as an additive group and it is known as the free abelian group of rank $n$. An element $v \in \mathbb{Z}^{n}$ is called an integer vector of size $n$ and can be written as a column vector $v=\left[v_{1}, \ldots, v_{n}\right]^{T}$. The standard basis of $\mathbb{Z}^{n}$ is $\left\{e_{1}, \ldots, e_{n}\right\}$ where $e_{i}=[0, \ldots, 1, \ldots, 0]^{T}(i=$ $1, \ldots, n)$ having 1 in the $i$-th position and 0 otherwise.

Denote the set of $n \times n$ matrices over $\mathbb{Z}$ by $M_{n}(\mathbb{Z})$. An unimodular matrix of size $n$ is an element $A \in M_{n}(\mathbb{Z})$ having determinant \pm 1 . All unimodular matrices of size $n$ form a group with the operation of matrix multiplication. It is known as the general linear group $G L_{n}(\mathbb{Z})$. That is to say

$$
G L_{n}(\mathbb{Z})=\left\{A \in M_{n}(\mathbb{Z}) \mid \operatorname{det} A= \pm 1\right\} .
$$

An element $A \in G L_{n}(\mathbb{Z})$ induces an automorphism of $\mathbb{Z}^{n}$ by

$$
\begin{aligned}
A: \quad \mathbb{Z}^{n} & \rightarrow \mathbb{Z}^{n} \\
v & \mapsto A v
\end{aligned}
$$

and in fact $G L_{n}(\mathbb{Z})$ is the automorphism group of $\mathbb{Z}^{n}$. The orbit of $v \in \mathbb{Z}^{n}$ by $A$ is the set $\left\{A^{k} v \mid k \in \mathbb{Z}\right\}$. It generates a subgroup of $\mathbb{Z}^{n}$ whose elements are integral linear combinations of finitely many elements in $\left\{A^{k} v \mid k \in \mathbb{Z}\right\}$.

\footnotetext{
* The authors thank the referees for their work and suggestions that have helped improve this paper.

${ }^{\dagger}$ The author is partially supported by National Natural Science Foundation of China (No. 11571246).

E-mail addresses: wujianchun@suda.edu.cn (Jianchun Wu), 20174207001@stu.suda.edu.cn (Yonghu Zheng)
} 
Definition 1.1. Let $S$ be a subset of $\mathbb{Z}^{n}$ and $A \in G L_{n}(\mathbb{Z})$. The orbit subgroup $O G_{A}(S)$ on $S$ by $A$ is the subgroup of $\mathbb{Z}^{n}$ generated by $\left\{A^{k} v \mid k \in \mathbb{Z}, v \in S\right\}$. If $O G_{A}(S)$ is the full group $\mathbb{Z}^{n}$, we call $S$ a generating orbit set of $A$.

Remark 1.2. If $S$ generates $\mathbb{Z}^{n}$, then it is a generating orbit set for any $A \in G L_{n}(\mathbb{Z})$ while any generating orbit set of the identity matrix $I_{n}$ must generate $\mathbb{Z}^{n}$.

We consider the question that if $S$ is a generating orbit set of a fixed element $A \in$ $G L_{n}(\mathbb{Z})$, how many elements must $S$ contain. Among all generating orbit sets of $A$ those having minimal cardinalities are called minimal generating orbit sets. So the question is:

Question 1.3. Let $A \in G L_{n}(\mathbb{Z})$, what is

$$
m_{A}=\min \left\{\# S \mid O G_{A}(S)=\mathbb{Z}^{n}\right\}
$$

the cardinality of a minimal generating orbit set of $A$ ?

In this paper, we determine $m_{A}$ and construct an explicit minimal generating orbit set for each torsion element (defined below) $A$ in $G L_{n}(\mathbb{Z})(n \leq 4)$.

Definition 1.4. $A \in G L_{n}(\mathbb{Z})$ is called a torsion element if $A^{m}$ is the identity matrix $I_{n}$ for some positive integer $m$. A torsion element $A$ has order $d$ if $d$ is the minimal positive integer such that $A^{d}=I_{n}$.

There are two other interpretations of $m_{A}$. The first one is from ring and module theory. Fixing $A \in G L_{n}(\mathbb{Z}), \mathbb{Z}^{n}$ can be viewed as a module over the ring of integral polynomials $\mathbb{Z}[X]$ by defining $p(X) \cdot v=p(A) v\left(p(x) \in \mathbb{Z}[X], v \in \mathbb{Z}^{n}\right)$. Denote the rank (i.e., the minimal number of generators) of the $\mathbb{Z}[X]$-module $\mathbb{Z}^{n}$ by $\operatorname{rk}_{\mathbb{Z}[X]}\left(\mathbb{Z}^{n}\right)$, then $m_{A}=\operatorname{rk}_{\mathbb{Z}[X]}\left(\mathbb{Z}^{n}\right)$. Since $A$ is invertible, we can also define $\mathbb{Z}^{n}$ as a module over the ring of integral Laurent polynomials $\mathbb{Z}\left[X, X^{-1}\right]$ by the same rule and $m_{A}$ is equal to the rank of the $\mathbb{Z}\left[X, X^{-1}\right]$-module $\mathbb{Z}^{n}$. The second one is from combinatorial group theory. Let $G=\mathbb{Z}^{n} \rtimes_{A} \mathbb{Z}$ be the semidirect product of $\mathbb{Z}^{n}$ and $\mathbb{Z}$ determined by $A$, the rank (i.e., the minimal number of generators) of $G$ is denoted by $\operatorname{rk}(G)$, then $m_{A}=\operatorname{rk}(G)-1$ (see [6, Corollary 2.4]).

It is obvious that $m_{A} \leq n$ since we can choose $S$ to be a basis of $\mathbb{Z}^{n} . m_{A}$ is computable for each $A \in G L_{2}(\mathbb{Z})$ ([6, Corollary 3.3]) because in this case $m_{A}=1$ or 2 and by Lemma 2.4 we know when $m_{A}=1$. To the authors' knowledge, it is not known whether $m_{A}$ is computable for each non-torsion element $A \in G L_{n}(\mathbb{Z})$ even when $n=3$. Another fact is that

Proposition 1.5. $m_{A}$ is a conjugacy invariant. That is to say $m_{X A X^{-1}}=m_{A}$ for any $X \in G L_{n}(\mathbb{Z})$.

Proof. If $S$ is a generating orbit set of $A$, then $\{X v \mid v \in S\}$ is a generating orbit set for $X A X^{-1}$ and has the same cardinality as $S$. So $m_{X A X^{-1}} \leq m_{A}$. For the same reason $m_{A}=m_{X^{-1} X A X^{-1} X} \leq m_{X A X^{-1}}$.

Remark 1.6. Throughout this paper, conjugation always means integral conjugation. This is to say, $B$ is conjugate to $A$ by $X$ if and only if $B=X A X^{-1}$ for some $X \in G L_{n}(\mathbb{Z})$. If the basis of $\mathbb{Z}^{n}$ is changed, an automorphism of $\mathbb{Z}^{n}$ may correspond to different matrices, but they are (integrally) conjugate to each other. 
The problem of the classification of conjugacy classes in $G L_{n}(\mathbb{Z})$ has long history and is not completely solved, see $[1,4,5,8]$. For more results about classifying torsion elements up to conjugacy, see $[7,9,10]$.

After introducing some facts in Section 2, we use classification results (Theorems 2.6, 2.8 and 2.10) from $[7,9,10]$ and construct a minimal generating orbit set for each representative of conjugacy class of torsion elements in Section 3. The results are listed in Table 1, Table 2 and Table 3 respectively.

\section{Preliminary}

Definition 2.1. The companion matrix of a monic polynomial $f(x)=a_{0}+a_{1} x+\cdots+$ $a_{n-1} x^{n-1}+x^{n}$ is the square matrix

$$
C(f)=\left[\begin{array}{ccccc}
0 & 0 & \ldots & 0 & -a_{0} \\
1 & 0 & \ldots & 0 & -a_{1} \\
0 & 1 & \ldots & 0 & -a_{2} \\
\vdots & \vdots & \ddots & \vdots & \vdots \\
0 & 0 & \ldots & 1 & -a_{n-1}
\end{array}\right] .
$$

Remark 2.2 ([3, p147]). The characteristic and minimal polynomials of a companion matrix do coincide.

Definition 2.3. The companion matrix of the characteristic polynomial of $A \in G L_{n}(\mathbb{Z})$ is called the companion of $A$.

The following observation is standard (see [6]), we prefer to write down a proof here by using our notations.

Lemma 2.4. Suppose $A \in G L_{n}(\mathbb{Z})$, then $m_{A}=1$ if and only if $A$ is conjugate to its companion.

Proof. Denote the characteristic polynomial of $A$ by $p(x)$ and the companion of $A$ by $C$. Note that $C=C(p)$.

If $m_{A}=1$, suppose $S=\{v\}$ is a minimal generating orbit set of $A$, then $\left\{A^{k} v \mid k \in \mathbb{Z}\right\}$ generates $\mathbb{Z}^{n}$. By Cayley-Hamilton theorem, for any $k \in \mathbb{Z}, A^{k} v$ is an integral linear combination of $\left\{v, A v, \ldots, A^{n-1} v\right\}$. That is to say $\left\{v, A v, \ldots, A^{n-1} v\right\}$ is a basis of $\mathbb{Z}^{n}$. The matrix of the automorphism $A: \mathbb{Z}^{n} \rightarrow \mathbb{Z}^{n}$ under this basis is the companion matrix $C(p)$ and so $A$ is conjugate to $C(p)$.

Conversely, if $A$ is conjugate to its companion $C=C(p)$, it is easy to check that $\left\{e_{1}, C e_{1}, \ldots, C^{n-1} e_{1}\right\}$ generates $\mathbb{Z}^{n}$ where $e_{1}=[1,0, \ldots, 0]^{T}$. So $m_{C}=1$ and $m_{A}=m_{C}$ by Proposition 1.5.

For convenience, suppose $A \in G L_{m}(\mathbb{Z}), B \in G L_{n}(\mathbb{Z})$, denote by $A \oplus B$ the matrix $\left[\begin{array}{cc}A & 0 \\ 0 & B\end{array}\right] \in G L_{m+n}(\mathbb{Z})$. Similarly, for $u \in \mathbb{Z}^{m}, v \in \mathbb{Z}^{n}$, the column vector $\left[\begin{array}{l}u \\ v\end{array}\right] \in$ $\mathbb{Z}^{m} \oplus \mathbb{Z}^{n}=\mathbb{Z}^{m+n}$ is denoted by $u \oplus v$. We have $(A \oplus B)(u \oplus v)=A u \oplus B v$. 
Lemma 2.5. (1) $m_{A}=m_{-A}$; (2) $\max \left\{m_{A}, m_{B}\right\} \leq m_{A \oplus B} \leq m_{A}+m_{B}$.

Proof. (1) $A$ and $-A$ have same generating orbit sets.

(2) For $A \in G L_{m}(\mathbb{Z}), B \in G L_{n}(\mathbb{Z})$, suppose $S$ is a minimal generating orbit set of $A \oplus B$ and each element $s \in S$ is written as $\left[\begin{array}{l}s_{A} \\ s_{B}\end{array}\right]$ where $s_{A} \in \mathbb{Z}^{m}, s_{B} \in \mathbb{Z}^{n}$. Then $S_{A}=\left\{s_{A} \mid s \in S\right\}$ is a generating orbit set for $A$ and so $m_{A} \leq\left|S_{A}\right| \leq|S|=m_{A \oplus B}$. Similarly, $m_{B} \leq m_{A \oplus B}$.

Suppose $S_{A}$ and $S_{B}$ are minimal generating orbit sets of $A \in G L_{m}(\mathbb{Z})$ and $B \in$ $G L_{n}(\mathbb{Z})$ respectively. Let $S=\left\{u \oplus 0 \mid u \in S_{A}\right\} \cup\left\{0 \oplus v \mid v \in S_{B}\right\} \subset \mathbb{Z}^{m+n}$. Then $S$ is a generating orbit set for $A \oplus B$ and $m_{A \oplus B} \leq|S|=\left|S_{A}\right|+\left|S_{B}\right|=m_{A}+m_{B}$.

Theorem 2.6 ([7], [8, Chapter IX], [10, Lemma 1.6]). Each torsion element in $G L_{2}(\mathbb{Z})$ is conjugate to one of the matrices listed in the second row of the table below where $K=\left[\begin{array}{cc}1 & 0 \\ 0 & -1\end{array}\right], U=\left[\begin{array}{cc}1 & 1 \\ 0 & -1\end{array}\right], W=\left[\begin{array}{cc}0 & -1 \\ 1 & -1\end{array}\right], J=\left[\begin{array}{cc}0 & -1 \\ 1 & 0\end{array}\right]$.

\begin{tabular}{|c|c|c|c|c|c|}
\hline Order & $d=1$ & $d=2$ & $d=3$ & $d=4$ & $d=6$ \\
\hline $\begin{array}{c}\text { Representative of } \\
\text { conjugacy class }\end{array}$ & $I_{2}$ & $-I_{2}, K, U$ & $W$ & $J$ & $-W$ \\
\hline
\end{tabular}

Remark 2.7. In some literatures, the representative $U$ is replaced by $V=\left[\begin{array}{ll}0 & 1 \\ 1 & 0\end{array}\right]$.

Theorem 2.8 ([9, page 173, 174, 184]). Each torsion element in $G L_{3}(\mathbb{Z})$ is conjugate to one of the matrices listed in the second column of the table below where $K=\left[\begin{array}{cc}1 & 0 \\ 0 & -1\end{array}\right], V=\left[\begin{array}{ll}0 & 1 \\ 1 & 0\end{array}\right], W=\left[\begin{array}{cc}0 & -1 \\ 1 & -1\end{array}\right], J=\left[\begin{array}{cc}0 & -1 \\ 1 & 0\end{array}\right], E_{1}=\left[\begin{array}{ll}0 & 1\end{array}\right]$.

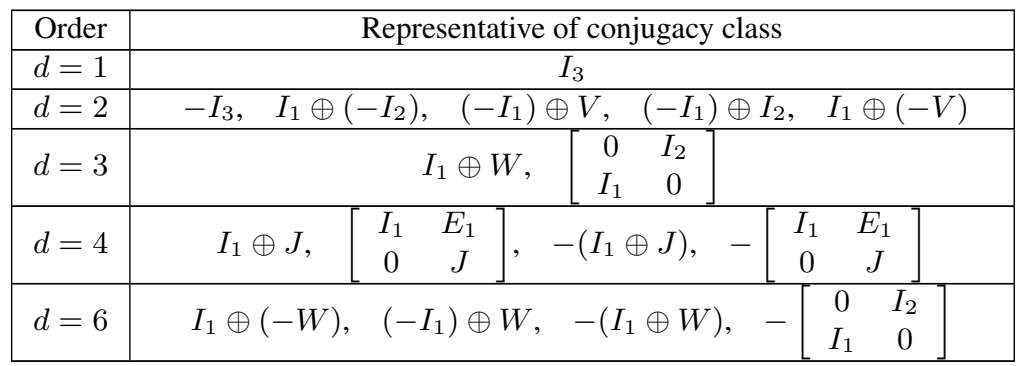

Remark 2.9. Since $\left[\begin{array}{cc}0 & -1 \\ 1 & 1\end{array}\right]$ is conjugate to $-W=\left[\begin{array}{cc}0 & 1 \\ -1 & 1\end{array}\right]$ by $\left[\begin{array}{cc}-1 & 1 \\ 1 & 0\end{array}\right]$, the order 6 element $W_{1}=\left[\begin{array}{ccc}1 & 0 & 0 \\ 0 & 0 & -1 \\ 0 & 1 & 1\end{array}\right]$ in [9, page 184] is conjugate to $I_{1} \oplus(-W)$ and we choose the latter as a representative for simplicity and replace $W_{2}$ (in the same page) by $\left(-I_{1}\right) \oplus W$ for the same reason. 
Theorem 2.10 ([10, page 492]). Each torsion element in $G L_{4}(\mathbb{Z})$ is conjugate to one of the matrices listed in the second column of the table below where $K=\left[\begin{array}{cc}1 & 0 \\ 0 & -1\end{array}\right]$, $U=\left[\begin{array}{cc}1 & 1 \\ 0 & -1\end{array}\right], W=\left[\begin{array}{cc}0 & -1 \\ 1 & -1\end{array}\right], J=\left[\begin{array}{cc}0 & -1 \\ 1 & 0\end{array}\right], E=\left[\begin{array}{ll}0 & 1 \\ 0 & 0\end{array}\right]$ and $C_{5}, C_{8}, C_{10}$, $C_{12}$ are the companion matrices of the cyclotomic polynomials $\Phi_{5}(x)=x^{4}+x^{3}+x^{2}+$ $x+1, \Phi_{8}(x)=x^{4}+1, \Phi_{10}(x)=x^{4}-x^{3}+x^{2}-x+1, \Phi_{12}(x)=x^{4}-x^{2}+1$ respectively.

\begin{tabular}{|c|c|}
\hline Order & Representative of conjugacy class \\
\hline$d=1$ & $I_{4}$ \\
\hline$d=2$ & $\begin{array}{c}-I_{4}, \quad K \oplus\left(-I_{2}\right), \quad U \oplus\left(-I_{2}\right) \\
I_{2} \oplus\left(-I_{2}\right), \quad K \oplus U, \quad U \oplus U, \\
I_{2} \oplus K, \quad I_{2} \oplus U\end{array}$ \\
\hline$d=3$ & $W \oplus W, \quad I_{2} \oplus W, \quad\left[\begin{array}{cc}I_{2} & E \\
0 & W\end{array}\right]$ \\
\hline$d=4$ & $\begin{array}{c}J \oplus J, \quad I_{2} \oplus J, \quad\left[\begin{array}{ll}I_{2} & E \\
0 & J\end{array}\right], \\
\left(-I_{2}\right) \oplus J, \quad\left[\begin{array}{cc}-I_{2} & E \\
0 & J\end{array}\right], \quad K \oplus J, \\
{\left[\begin{array}{cc}K & E \\
0 & J\end{array}\right], \quad\left[\begin{array}{cc}K & I_{2} \\
0 & J\end{array}\right], \quad\left[\begin{array}{cc}K & I_{2}-E \\
0 & J\end{array}\right],} \\
U \oplus J, \quad\left[\begin{array}{cc}U & E \\
0 & J\end{array}\right], \quad\left[\begin{array}{cc}U & I \\
0 & J\end{array}\right]\end{array}$ \\
\hline$d=5$ & $C_{5}$ \\
\hline$d=6$ & $\begin{array}{c}-(W \oplus W), \quad I_{2} \oplus(-W), \quad\left(-I_{2}\right) \oplus W, \\
-\left(I_{2} \oplus W\right), \quad\left[\begin{array}{cc}-I_{2} & E \\
0 & -W\end{array}\right], \quad K \oplus W, \\
{\left[\begin{array}{cc}K & E \\
0 & W\end{array}\right], \quad U \oplus W, \quad\left[\begin{array}{cc}U & E \\
0 & W\end{array}\right],} \\
-(K \oplus W), \quad\left[\begin{array}{cc}-K & E \\
0 & -W\end{array}\right], \quad-(U \oplus W), \\
{\left[\begin{array}{cc}-U & E \\
0 & -W\end{array}\right], \quad W \oplus(-W), \quad\left[\begin{array}{cc}W & E \\
0 & -W\end{array}\right]}\end{array}$ \\
\hline$d=8$ & $C_{8}$ \\
\hline$d=10$ & $C_{10}$ \\
\hline$d=12$ & $C_{12}, \quad J \oplus W, \quad J \oplus(-W)$ \\
\hline
\end{tabular}

\section{Constructing minimal generating orbit sets}

In this section, we determine $m_{A}$ and construct a minimal generating orbit set $S_{A}$ for $A$ being a representative of conjugacy class in $G L_{n}(\mathbb{Z})(n \leq 4)$. Results for other torsion elements can be obtained as follows:

Given a torsion element $B$ in $G L_{n}(\mathbb{Z})(n \leq 4)$, then $B$ is conjugate to some representative $A$ listed in Theorem 2.6, 2.8, 2.10 by some $X \in G L_{n}(\mathbb{Z})$. There is an algorithm for deciding whether two elements in $G L_{n}(\mathbb{Z})$ are conjugate.

Theorem 3.1 ([2, Theorem A]). Given two matrices $A, B \in G L_{n}(\mathbb{Z})$, there is an algorithm to deciding whether there exists a matrix $X \in G L_{n}(\mathbb{Z})$ such that $B=X A X^{-1}$. If 
the answer is "yes" the algorithm constructs a conjugating matrix $X$.

So we can determine $A$ and construct $X$ at the same time by the algorithm from Theorem 3.1 through enumerating $A$ in the lists. Now $m_{B}=m_{A}$ and a minimal generating orbit set for $B$ can be obtained as $\left\{X v \mid v \in S_{A}\right\}$.

\subsection{Conjugacy classes of companion matrices}

In Theorem 2.6, 2.8 and 2.10, the representatives of conjugacy classes of torsion elements in the tables are not always companion matrices. The algorithm in Theorem 3.1 for deciding whether $A \in G L_{n}(\mathbb{Z})$ is conjugate to its companion is hard to be conducted by hand. But for torsion elements, especially when $n$ is small, there is a simpler method to handle most cases. We describe it as the following three steps.

Step 1: Compute the characteristic polynomial and minimal polynomial of $A$, if they are not the same, then $A$ is not conjugate to its companion, moreover $m_{A} \geq 2$, otherwise go to Step 2.

Step 2: If there is only one representative with the same characteristic and minimal polynomials as those of $A$ in the table, then $A$ is conjugate to its companion because the companion of $A$ is also a torsion element and has the same characteristic and minimal polynomials, otherwise go to Step 3 .

Step 3: If we can find a minimal generating orbit set with only one element for $A$, then by Lemma $2.4 A$ is conjugate to its companion, otherwise we apply the algorithm in Theorem 3.1.

\subsection{Direct sum}

We already know $m_{A \oplus B} \leq m_{A}+m_{B}$ by Lemma 2.5 , the equality is not always true, for instance:

Example 3.2. Suppose $W=\left[\begin{array}{ll}0 & -1 \\ 1 & -1\end{array}\right]$, the companion matrix of $f(x)=x^{2}+x+1$, so $m_{W}=1$ and by Lemma $2.5, m_{-W}=1$. Now for $I_{1} \oplus(-W)=\left[\begin{array}{ccc}1 & 0 & 0 \\ 0 & 0 & 1 \\ 0 & -1 & 1\end{array}\right]$, one can check $\left\{[1,1,0]^{T}\right\}$ is a minimal generating orbit set. Another example is shown in Example 3.9.

But for some special cases the equality can be obtained. We have:

Proposition 3.3. If $m_{A}=1$ then $m_{A \oplus A}=2$.

Proof. By Lemma 2.5, $m_{A \oplus A} \leq 2$. The characteristic polynomial and minimal polynomial of $A \oplus A$ are not the same, so $A \oplus A$ is not conjugate to its companion and $m_{A \oplus A} \geq 2$ by Lemma 2.4 .

\subsection{Construction}

Let $e_{1}=[1,0,0,0]^{T}, e_{2}=[0,1,0,0]^{T}, e_{3}=[0,0,1,0]^{T}, e_{4}=[0,0,0,1]^{T}$ be the standard basis elements of $\mathbb{Z}^{4}$. 
Proposition 3.4. Suppose $A=I_{2} \oplus W=\left[\begin{array}{rrrr}1 & 0 & 0 & 0 \\ 0 & 1 & 0 & 0 \\ 0 & 0 & 0 & -1 \\ 0 & 0 & 1 & -1\end{array}\right]$, then $m_{A}=3$.

Proof. Suppose some generating orbit set of $A$ contains only $u=\left[u_{1}, u_{2}, u_{3}, u_{4}\right]^{T}$ and $v=$ $\left[v_{1}, v_{2}, v_{3}, v_{4}\right]^{T}$, that is to say, $\left\{A^{k} u, A^{k} v \mid k \in \mathbb{Z}\right\}$ generates $\mathbb{Z}^{4}$. By Cayley-Hamilton theorem, for any $k \in \mathbb{Z}, w \in \mathbb{Z}^{4}, A^{k} w$ is an integral linear combination of $w, A w, A^{2} w, A^{3} w$. Since $A^{3}=I_{4}$, we have $\left\langle u, A u, A^{2} u, v, A v, A^{2} v\right\rangle=\mathbb{Z}^{4}$.

Note that $A^{2}=\left[\begin{array}{rrrr}1 & 0 & 0 & 0 \\ 0 & 1 & 0 & 0 \\ 0 & 0 & -1 & 1 \\ 0 & 0 & -1 & 0\end{array}\right]$ so

$\left\langle\left[\begin{array}{l}u_{1} \\ u_{2} \\ u_{3} \\ u_{4}\end{array}\right],\left[\begin{array}{c}u_{1} \\ u_{2} \\ -u_{4} \\ u_{3}-u_{4}\end{array}\right],\left[\begin{array}{c}u_{1} \\ u_{2} \\ -u_{3}+u_{4} \\ -u_{3}\end{array}\right],\left[\begin{array}{c}v_{1} \\ v_{2} \\ v_{3} \\ v_{4}\end{array}\right],\left[\begin{array}{c}v_{1} \\ v_{2} \\ -v_{4} \\ v_{3}-v_{4}\end{array}\right],\left[\begin{array}{c}v_{1} \\ v_{2} \\ -v_{3}+v_{4} \\ -v_{3}\end{array}\right]\right\rangle=\mathbb{Z}^{4}$,

if and only if

$$
\left\langle\left[\begin{array}{l}
u_{1} \\
u_{2} \\
u_{3} \\
u_{4}
\end{array}\right],\left[\begin{array}{c}
0 \\
0 \\
u_{3}+u_{4} \\
2 u_{4}-u_{3}
\end{array}\right],\left[\begin{array}{c}
0 \\
0 \\
2 u_{3}-u_{4} \\
u_{4}+u_{3}
\end{array}\right],\left[\begin{array}{c}
v_{1} \\
v_{2} \\
v_{3} \\
v_{4}
\end{array}\right],\left[\begin{array}{c}
0 \\
0 \\
v_{3}+v_{4} \\
2 v_{4}-v_{3}
\end{array}\right],\left[\begin{array}{c}
0 \\
0 \\
2 v_{3}-v_{4} \\
v_{4}+v_{3}
\end{array}\right]\right\rangle=\mathbb{Z}^{4},
$$

if and only if

$$
\left\langle\left[\begin{array}{l}
u_{1} \\
u_{2} \\
u_{3} \\
u_{4}
\end{array}\right],\left[\begin{array}{c}
0 \\
0 \\
u_{3}+u_{4} \\
2 u_{4}-u_{3}
\end{array}\right],\left[\begin{array}{c}
0 \\
0 \\
3 u_{3} \\
3 u_{4}
\end{array}\right],\left[\begin{array}{c}
v_{1} \\
v_{2} \\
v_{3} \\
v_{4}
\end{array}\right],\left[\begin{array}{c}
0 \\
0 \\
v_{3}+v_{4} \\
2 v_{4}-v_{3}
\end{array}\right],\left[\begin{array}{c}
0 \\
0 \\
3 v_{3} \\
3 v_{4}
\end{array}\right]\right\rangle=\mathbb{Z}^{4},
$$

if and only if

$$
\left[\begin{array}{ll}
u_{1} & v_{1} \\
u_{2} & v_{2}
\end{array}\right] \in G L_{2}(\mathbb{Z}) \text { and }\left\{\left[\begin{array}{c}
u_{3}+u_{4} \\
2 u_{4}-u_{3}
\end{array}\right],\left[\begin{array}{c}
3 u_{3} \\
3 u_{4}
\end{array}\right],\left[\begin{array}{c}
v_{3}+v_{4} \\
2 v_{4}-v_{3}
\end{array}\right],\left[\begin{array}{c}
3 v_{3} \\
3 v_{4}
\end{array}\right]\right\}
$$

generates $\mathbb{Z}^{2}$.

We take $\bmod 3$, then $\left\{\left[\begin{array}{c}u_{3}+u_{4} \\ 2 u_{4}-u_{3}\end{array}\right],\left[\begin{array}{c}v_{3}+v_{4} \\ 2 v_{4}-v_{3}\end{array}\right]\right\}$ generates $\mathbb{Z}_{3}^{2}$. It is impossible because the determinant of $\left[\begin{array}{cc}u_{3}+u_{4} & v_{3}+v_{4} \\ 2 u_{4}-u_{3} & 2 v_{4}-v_{3}\end{array}\right]$ is $0 \bmod 3$.

We have proved that $m_{A}>2$. One can check $S=\left\{e_{1}, e_{2}, e_{4}\right\}$ is a generating orbit set for $A$. So $S$ is minimal and $m_{A}=3$.

Remark 3.5. Suppose $A=\left(-I_{m}\right) \oplus I_{n-m} \in G L_{n}(\mathbb{Z})$, then $m_{A}=n$. More or less this fact is trivial, it can be proved by using similar method for proving Proposition 3.4. 


\subsection{Summary}

Now we can determine $m_{A}$ for every torsion element $A \in G L_{n}(\mathbb{Z})(n \leq 4)$ by using methods discussed in Section 3.1, 3.2, 3.3. We summarize them as the following reasons:

Trivial: For some trivial cases, we have $m_{A}=n$, for instance $A=I_{n}, A=\left(-I_{m}\right) \oplus$ $I_{n-m}$ and so on.

R1: $A$ is conjugate to its companion or we can find a generating orbit set for $A$ with only one element, so $m_{A}=1$. It can be done through the three steps in Section 3.1.

R2: $A$ is not conjugate to its companion and we can find a generating orbit set for $A$ with two elements, so $m_{A}=2$. It can be done through steps in Section 3.1 and for some special cases by Proposition 3.3.

R3: We can prove $m_{A}>2$ and find a generating orbit set for $A$ with three elements, so $m_{A}=3$. We have shown how to do it through an example by Proposition 3.4 in Section 3.3. The argument there can be applied to similar cases.

We will show part of the procedure by Example 3.9, 3.10 after the statements of main results in Section 3.5.

\subsection{Determination of $m_{A}$ for torsion elements in $G L_{n}(\mathbb{Z})(n \leq 4)$}

\subsubsection{Torsion elements in $G L_{2}(\mathbb{Z})$}

The representatives of conjugacy classes of torsion elements in $G L_{2}(\mathbb{Z})$ are already listed in Theorem 2.6. It is easy to find minimal generating orbit sets for these elements and we have

Theorem 3.6. For a given torsion element $A \in G L_{2}(\mathbb{Z}), m_{A}$ is determined and a minimal generating orbit set is constructed explicitly. The results are listed in Table 1 where $K=\left[\begin{array}{cc}1 & 0 \\ 0 & -1\end{array}\right], U=\left[\begin{array}{cc}1 & 1 \\ 0 & -1\end{array}\right], W=\left[\begin{array}{cc}0 & -1 \\ 1 & -1\end{array}\right], J=\left[\begin{array}{cc}0 & -1 \\ 1 & 0\end{array}\right], e_{1}=[1,0]^{T}$, $e_{2}=[0,1]^{T}$.

Table 1: Minimal generating orbit sets for torsion elements in $G L_{2}(\mathbb{Z})$

\begin{tabular}{|c|c|c|c|c|c|}
\hline \multirow{2}{*}{ Order } & $\begin{array}{c}\text { Representative of } \\
\text { Conjugacy Class }\end{array}$ & $m_{A}$ & $\begin{array}{c}\text { Minimal } \\
\text { Generating } \\
\text { Orbit Set }\end{array}$ & $\begin{array}{c}\text { Characteristic polynomial; } \\
\text { Minimal Polynomial }\end{array}$ & Reason \\
\hline $\mathrm{d}=1$ & $I_{2}$ & 2 & $\left\{e_{1}, e_{2}\right\}$ & $(x-1)^{2} ; x-1$ & Trivial \\
\hline $\mathrm{d}=2$ & $-I_{2}$ & 2 & $\left\{e_{1}, e_{2}\right\}$ & $(x+1)^{2} ; x+1$ & Trivial \\
\cline { 2 - 7 } & $K=I_{1} \oplus\left(-I_{1}\right)$ & 2 & $\left\{e_{1}, e_{2}\right\}$ & $x^{2}-1 ; x^{2}-1$ & Trivial \\
\cline { 2 - 7 } & $U$ & 1 & $\left\{e_{2}\right\}$ & $x^{2}-1 ; x^{2}-1$ & R1 \\
\hline $\mathrm{d}=3$ & $W$ & 1 & $\left\{e_{1}\right\}$ & $x^{2}+x+1 ; x^{2}+x+1$ & $\mathrm{R} 1$ \\
\hline $\mathrm{d}=4$ & $J$ & 1 & $\left\{e_{1}\right\}$ & $x^{2}+1 ; x^{2}+1$ & $\mathrm{R} 1$ \\
\hline $\mathrm{d}=6$ & $-W$ & 1 & $\left\{e_{1}\right\}$ & $x^{2}-x+1 ; x^{2}-x+1$ & $\mathrm{R} 1$ \\
\hline
\end{tabular}

In Table 1 (also Table 2 and 3 below), the representatives of conjugacy classes of torsion 
elements are listed in the second column and their orders in the first column, $m_{A}$ and explicit minimal generating orbit sets are listed in the third and fourth columns respectively.

For the convenience of checking the results through the procedure in Section 3.4, we record the characteristic and minimal polynomials in the fifth column and the reasons for determining $m_{A}$ in the last column.

\subsubsection{Torsion elements in $G L_{3}(\mathbb{Z})$}

The representatives of conjugacy classes of torsion elements in $G L_{3}(\mathbb{Z})$ are already listed in Theorem 2.8 and we have

Theorem 3.7. For a given torsion element $A \in G L_{3}(\mathbb{Z}), m_{A}$ is determined and a minimal generating orbit set is constructed explicitly. The results are listed in Table 2 where $K=\left[\begin{array}{cc}1 & 0 \\ 0 & -1\end{array}\right], V=\left[\begin{array}{ll}0 & 1 \\ 1 & 0\end{array}\right], W=\left[\begin{array}{cc}0 & -1 \\ 1 & -1\end{array}\right], J=\left[\begin{array}{cc}0 & -1 \\ 1 & 0\end{array}\right], E_{1}=\left[\begin{array}{ll}0 & 1\end{array}\right]$, $e_{1}=[1,0,0]^{T}, e_{2}=[0,1,0]^{T}, e_{3}=[0,0,1]^{T}$.

Table 2: Minimal generating orbit sets for torsion elements in $G L_{3}(\mathbb{Z})$

\begin{tabular}{|c|c|c|c|c|c|}
\hline Order & $\begin{array}{l}\text { Representative of } \\
\text { Conjugacy Class }\end{array}$ & $m_{A}$ & $\begin{array}{c}\text { Minimal } \\
\text { Generating } \\
\text { Orbit Set }\end{array}$ & $\begin{array}{l}\text { Characteristic polynomial; } \\
\text { Minimal Polynomial }\end{array}$ & Reason \\
\hline $\mathrm{d}=1$ & $I_{3}$ & 3 & $\left\{e_{1}, e_{2}, e_{3}\right\}$ & $(x-1)^{3} ; x-1$ & Trivial \\
\hline \multirow[t]{5}{*}{$\mathrm{d}=2$} & $-I_{3}$ & 3 & $\left\{e_{1}, e_{2}, e_{3}\right\}$ & $(x+1)^{3} ; x+1$ & Trivial \\
\hline & $I_{1} \oplus\left(-I_{2}\right)$ & 3 & $\left\{e_{1}, e_{2}, e_{3}\right\}$ & $x^{3}+x^{2}-x-1 ; x^{2}-1$ & Trivial \\
\hline & $\left(-I_{1}\right) \oplus V$ & 2 & $\left\{e_{1}, e_{2}\right\}$ & $x^{3}+x^{2}-x-1 ; x^{2}-1$ & $\mathrm{R} 2$ \\
\hline & $\left(-I_{1}\right) \oplus I_{2}$ & 3 & $\left\{e_{1}, e_{2}, e_{3}\right\}$ & $x^{3}-x^{2}-x+1 ; x^{2}-1$ & Trivial \\
\hline & $I_{1} \oplus(-V)$ & 2 & $\left\{e_{1}, e_{2}\right\}$ & $x^{3}-x^{2}-x+1 ; x^{2}-1$ & $\mathrm{R} 2$ \\
\hline \multirow[t]{2}{*}{$d=3$} & $I_{1} \oplus W$ & 2 & $\left\{e_{1}, e_{2}\right\}$ & $x^{3}-1 ; x^{3}-1$ & $\mathrm{R} 2$ \\
\hline & $\begin{array}{cc}0 & I_{2} \\
I_{1} & 0\end{array}$ & 1 & $\left\{e_{1}\right\}$ & $x^{3}-1 ; x^{3}-1$ & $\mathrm{R} 1$ \\
\hline \multirow[t]{4}{*}{$\mathrm{d}=4$} & $I_{1} \oplus J$ & 2 & $\left\{e_{1}, e_{3}\right\}$ & $\begin{array}{c}x^{3}-x^{2}+x-1 \\
x^{3}-x^{2}+x-1\end{array}$ & $\mathrm{R} 2$ \\
\hline & {$\left[\begin{array}{cc}I_{1} & E_{1} \\
0 & J\end{array}\right]$} & 1 & $\left\{e_{2}\right\}$ & $\begin{array}{c}x^{3}-x^{2}+x-1 \\
x^{3}-x^{2}+x-1\end{array}$ & $\mathrm{R} 1$ \\
\hline & $\left(-I_{1}\right) \oplus J$ & 2 & $\left\{e_{1}, e_{3}\right\}$ & $\begin{array}{c}x^{3}+x^{2}+x+1 \\
x^{3}+x^{2}+x+1\end{array}$ & $\mathrm{R} 2$ \\
\hline & $-\left[\begin{array}{cc}I_{1} & E_{1} \\
0 & J\end{array}\right]$ & 1 & $\left\{e_{2}\right\}$ & $\begin{array}{c}x^{3}+x^{2}+x+1 \\
x^{3}+x^{2}+x+1\end{array}$ & $\mathrm{R} 1$ \\
\hline \multirow[t]{2}{*}{$d=6$} & $I_{1} \oplus(-W)$ & 1 & $\left\{e_{1}+e_{2}\right\}$ & $\begin{array}{c}x^{3}-2 x^{2}+2 x-1 \\
x^{3}-2 x^{2}+2 x-1\end{array}$ & $\mathrm{R} 1$ \\
\hline & $\left(-I_{1}\right) \oplus W$ & 1 & $\left\{e_{1}+e_{2}\right\}$ & $\begin{array}{c}x^{3}+2 x^{2}+2 x+1 \\
x^{3}+2 x^{2}+2 x+1\end{array}$ & $\mathrm{R} 1$ \\
\hline
\end{tabular}




\begin{tabular}{|c|c|c|c|c|c|}
\hline Order & $\begin{array}{l}\text { Representative of } \\
\text { Conjugacy Class }\end{array}$ & $m_{A}$ & $\begin{array}{c}\text { Minimal } \\
\text { Generating } \\
\text { Orbit Set }\end{array}$ & $\begin{array}{l}\text { Characteristic polynomial } \\
\text { Minimal Polynomial }\end{array}$ & Reason \\
\hline & $-\left(I_{1} \oplus W\right)$ & 2 & $\left\{e_{1}, e_{2}\right\}$ & $x^{3}+1 ; x^{3}+1$ & $\mathrm{R} 2$ \\
\hline & $-\left[\begin{array}{cc}0 & I_{2} \\
I_{1} & 0\end{array}\right.$ & 1 & $\left\{e_{1}\right\}$ & $x^{3}+1 ; x^{3}+1$ & $\mathrm{R} 1$ \\
\hline
\end{tabular}

\subsubsection{Torsion elements in $G L_{4}(\mathbb{Z})$}

The representatives of conjugacy classes of torsion elements in $G L_{4}(\mathbb{Z})$ are already listed in Theorem 2.10 and we have

Theorem 3.8. For a given torsion element $A \in G L_{4}(\mathbb{Z}), m_{A}$ is determined and a minimal generating orbit set is constructed explicitly. The results are listed in Table 3 where $K=\left[\begin{array}{cc}1 & 0 \\ 0 & -1\end{array}\right], U=\left[\begin{array}{cc}1 & 1 \\ 0 & -1\end{array}\right], W=\left[\begin{array}{cc}0 & -1 \\ 1 & -1\end{array}\right], J=\left[\begin{array}{cc}0 & -1 \\ 1 & 0\end{array}\right], C_{5}, C_{8}, C_{10}$, $C_{12}$ are the companion matrices of the cyclotomic polynomials $\Phi_{5}(x)=x^{4}+x^{3}+x^{2}+$ $x+1, \Phi_{8}(x)=x^{4}+1, \Phi_{10}(x)=x^{4}-x^{3}+x^{2}-x+1, \Phi_{12}(x)=x^{4}-x^{2}+1$ respectively and $e_{1}=[1,0,0,0]^{T}, e_{2}=[0,1,0,0]^{T}, e_{3}=[0,0,1,0]^{T}, e_{4}=[0,0,0,1]^{T}$.

Table 3: Minimal generating orbit sets for torsion elements in $G L_{4}(\mathbb{Z})$

\begin{tabular}{|c|c|c|c|c|c|}
\hline Order & $\begin{array}{l}\text { Representative of } \\
\text { Conjugacy Class }\end{array}$ & $m_{A}$ & $\begin{array}{l}\text { Minimal } \\
\text { Generating } \\
\text { Orbit Set }\end{array}$ & $\begin{array}{l}\text { Characteristic polynomial } \\
\text { Minimal Polynomial }\end{array}$ & Reason \\
\hline $\mathrm{d}=1$ & $I_{4}$ & 4 & $\left\{e_{1}, e_{2}, e_{3}, e_{4}\right\}$ & $(x-1)^{4} ; x-1$ & Trivial \\
\hline \multirow[t]{8}{*}{$d=2$} & $-I_{4}$ & 4 & $\left\{e_{1}, e_{2}, e_{3}, e_{4}\right\}$ & $(x+1)^{4} ; x+1$ & Trivial \\
\hline & $K \oplus\left(-I_{2}\right)$ & 4 & $\left\{e_{1}, e_{2}, e_{3}, e_{4}\right\}$ & $\begin{array}{c}x^{4}+2 x^{3}-2 x-1 \\
x^{2}-1\end{array}$ & Trivial \\
\hline & $U \oplus\left(-I_{2}\right)$ & 3 & $\left\{e_{2}, e_{3}, e_{4}\right\}$ & $\begin{array}{c}x^{4}+2 x^{3}-2 x-1 \\
x^{2}-1\end{array}$ & $\mathrm{R} 3$ \\
\hline & $I_{2} \oplus\left(-I_{2}\right)$ & 4 & $\left\{e_{1}, e_{2}, e_{3}, e_{4}\right\}$ & $x^{4}-2 x^{2}+1 ; x^{2}-1$ & Trivial \\
\hline & $K \oplus U$ & 3 & $\left\{e_{1}, e_{2}, e_{4}\right\}$ & $x^{4}-2 x^{2}+1 ; x^{2}-1$ & $\mathrm{R} 3$ \\
\hline & $U \oplus U$ & 2 & $\left\{e_{2}, e_{4}\right\}$ & $x^{4}-2 x^{2}+1 ; x^{2}-1$ & $\mathrm{R} 2$ \\
\hline & $I_{2} \oplus K$ & 4 & $\left\{e_{1}, e_{2}, e_{3}, e_{4}\right\}$ & $\begin{array}{c}x^{4}-2 x^{3}+2 x-1 \\
x^{2}-1\end{array}$ & Trivial \\
\hline & $I_{2} \oplus U$ & 3 & $\left\{e_{1}, e_{2}, e_{4}\right\}$ & $\begin{array}{c}x^{4}-2 x^{3}+2 x-1 \\
x^{2}-1\end{array}$ & R3 \\
\hline \multirow[t]{2}{*}{$d=3$} & $W \oplus W$ & 2 & $\left\{e_{1}, e_{3}\right\}$ & $\begin{array}{c}x^{4}+2 x^{3}+3 x^{2}+2 x+1 \\
x^{2}+x+1\end{array}$ & $\mathrm{R} 2$ \\
\hline & $I_{2} \oplus W$ & 3 & $\left\{e_{1}, e_{2}, e_{4}\right\}$ & $\begin{array}{c}x^{4}-x^{3}-x+1 \\
x^{3}-1\end{array}$ & $\mathrm{R} 3$ \\
\hline
\end{tabular}




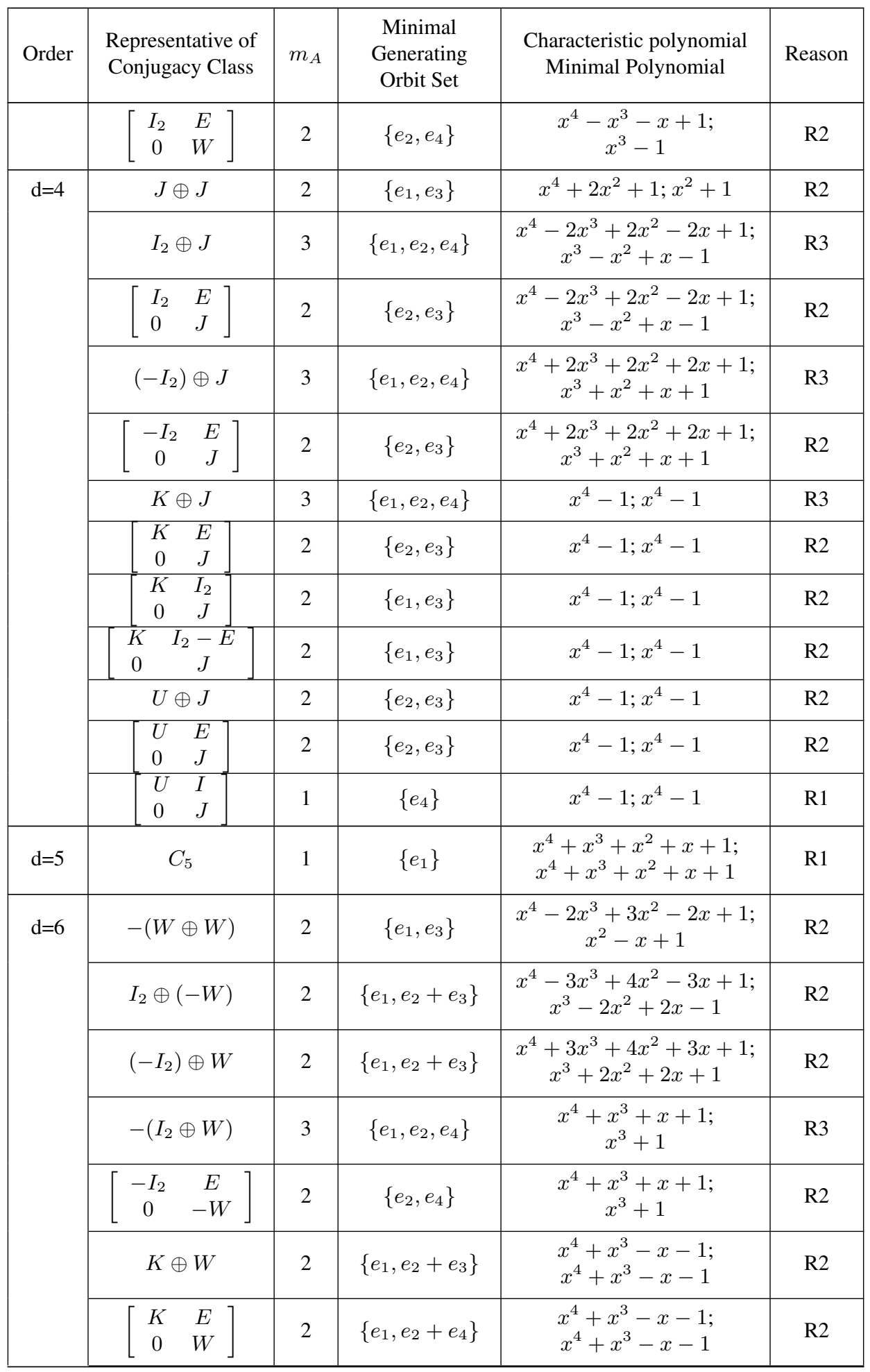




\begin{tabular}{|c|c|c|c|c|c|}
\hline Order & $\begin{array}{l}\text { Representative of } \\
\text { Conjugacy Class }\end{array}$ & $m_{A}$ & $\begin{array}{l}\text { Minimal } \\
\text { Generating } \\
\text { Orbit Set }\end{array}$ & $\begin{array}{l}\text { Characteristic polynomial } \\
\text { Minimal Polynomial }\end{array}$ & Reason \\
\hline & $U \oplus W$ & 2 & $\left\{e_{2}, e_{3}\right\}$ & $\begin{array}{c}x^{4}+x^{3}-x-1 \\
x^{4}+x^{3}-x-1\end{array}$ & $\mathrm{R} 2$ \\
\hline & {$\left[\begin{array}{cc}U & E \\
0 & W\end{array}\right]$} & 1 & $\left\{e_{2}-e_{3}\right\}$ & $\begin{array}{c}x^{4}+x^{3}-x-1 \\
x^{4}+x^{3}-x-1\end{array}$ & $\mathrm{R} 1$ \\
\hline & $-(K \oplus W)$ & 2 & $\left\{e_{1}, e_{2}+e_{3}\right\}$ & $\begin{array}{c}x^{4}-x^{3}+x-1 \\
x^{4}-x^{3}+x-1\end{array}$ & $\mathrm{R} 2$ \\
\hline & {$\left[\begin{array}{cc}-K & E \\
0 & -W\end{array}\right]$} & 2 & $\left\{e_{2}, e_{4}\right\}$ & $\begin{array}{c}x^{4}-x^{3}+x-1 \\
x^{4}-x^{3}+x-1\end{array}$ & $\mathrm{R} 2$ \\
\hline & $-(U \oplus W)$ & 2 & $\left\{e_{2}, e_{3}\right\}$ & $\begin{array}{c}x^{4}-x^{3}+x-1 \\
x^{4}-x^{3}+x-1\end{array}$ & $\mathrm{R} 2$ \\
\hline & {$\left[\begin{array}{cc}-U & E \\
0 & -W\end{array}\right]$} & 1 & $\left\{e_{2}+e_{3}\right\}$ & $\begin{array}{c}x^{4}-x^{3}+x-1 ; \\
x^{4}-x^{3}+x-1\end{array}$ & $\mathrm{R} 1$ \\
\hline & $W \oplus(-W)$ & 2 & $\left\{e_{1}, e_{3}\right\}$ & $\begin{array}{c}x^{4}+x^{2}+1 \\
x^{4}+x^{2}+1\end{array}$ & $\mathrm{R} 2$ \\
\hline & {$\left[\begin{array}{cc}W & E \\
0 & -W\end{array}\right]$} & 1 & $\left\{e_{4}\right\}$ & $\begin{array}{c}x^{4}+x^{2}+1 \\
x^{4}+x^{2}+1\end{array}$ & $\mathrm{R} 1$ \\
\hline $\mathrm{d}=8$ & $C_{8}$ & 1 & $\left\{e_{1}\right\}$ & $x^{4}+1 ; x^{4}+1$ & $\mathrm{R} 1$ \\
\hline $\mathrm{d}=10$ & $C_{10}$ & 1 & $\left\{e_{1}\right\}$ & $\begin{array}{c}x^{4}-x^{3}+x^{2}-x+1 \\
x^{4}-x^{3}+x^{2}-x+1\end{array}$ & $\mathrm{R} 1$ \\
\hline \multirow[t]{3}{*}{$\mathrm{d}=12$} & $C_{12}$ & 1 & $\left\{e_{1}\right\}$ & $\begin{array}{c}x^{4}-x^{2}+1 \\
x^{4}-x^{2}+1\end{array}$ & $\mathrm{R} 1$ \\
\hline & $J \oplus W$ & 1 & $\left\{e_{1}+e_{3}\right\}$ & $\begin{array}{c}x^{4}+x^{3}+2 x^{2}+x+1 \\
x^{4}+x^{3}+2 x^{2}+x+1\end{array}$ & $\mathrm{R} 1$ \\
\hline & $J \oplus(-W)$ & 1 & $\left\{e_{1}+e_{3}\right\}$ & $\begin{array}{c}x^{4}-x^{3}+2 x^{2}-x+1 \\
x^{4}-x^{3}+2 x^{2}-x+1\end{array}$ & $\mathrm{R} 1$ \\
\hline
\end{tabular}

Example 3.9. The last row in Table 3 is an order 12 representative

$$
J \oplus(-W)=\left[\begin{array}{rrrr}
0 & -1 & 0 & 0 \\
1 & 0 & 0 & 0 \\
0 & 0 & 0 & 1 \\
0 & 0 & -1 & 1
\end{array}\right]
$$

its characteristic polynomial and minimal polynomial are both $x^{4}-x^{3}+2 x^{2}-x+1$, so it may be conjugate to its companion. But there are no other elements in Table 3 with the same characteristic and minimal polynomials as those of $J \oplus(-W)$, so it must be conjugate to its companion. By Lemma 2.4, $J \oplus(-W)$ has a minimal generating orbit set with only one element and we can easily find it. 
Example 3.10. In Table $3, A=\left[\begin{array}{ll}U & I \\ 0 & J\end{array}\right]=\left[\begin{array}{rrrr}1 & 1 & 1 & 0 \\ 0 & -1 & 0 & 1 \\ 0 & 0 & 0 & -1 \\ 0 & 0 & 1 & 0\end{array}\right]$ is of order 4, the characteristic polynomial and minimal polynomial are both $x^{4}-1$, but other representatives (including $K \oplus J, U \oplus J$ and so on) have the same characteristic and minimal polynomials $x^{4}-1$, so we do Step 3 in Section 3.1 and find the orbit of $\left\{e_{4}=[0,0,0,1]^{T}\right\}$ by $A$ generates $\mathbb{Z}^{4}$ :

$$
\left\langle e_{4}, A e_{4}, A^{2} e_{4}, A^{3} e_{4}\right\rangle=\left\langle\left[\begin{array}{l}
0 \\
0 \\
0 \\
1
\end{array}\right],\left[\begin{array}{r}
0 \\
1 \\
-1 \\
0
\end{array}\right],\left[\begin{array}{r}
0 \\
-1 \\
0 \\
-1
\end{array}\right],\left[\begin{array}{r}
-1 \\
0 \\
1 \\
0
\end{array}\right]\right\rangle=\mathbb{Z}^{4}
$$

This is to say, $A$ is conjugate to its companion, but we don't need this fact since we already found a minimal generating orbit set.

\section{ORCID iDs}

Jianchun Wu (D) https://orcid.org/0000-0002-0802-5291

Yonghu Zheng (D) https://orcid.org/0000-0002-8152-8100

\section{References}

[1] E. C. Dade, The maximal finite groups of $4 \times 4$ integral matrices, Illinois J. Math. 9 (1965), 99-122, doi:10.1215/ijm/1256067584.

[2] F. J. Grunewald, Solution of the conjugacy problem in certain arithmetic groups, in: Word problems, II (Conf. on Decision Problems in Algebra, Oxford, 1976), North-Holland, AmsterdamNew York, volume 95 of Stud. Logic Foundations Math., pp. 101-139, 1980, doi:10.1016/ s0049-237x(08)71335-1.

[3] R. A. Horn and C. R. Johnson, Matrix analysis, Cambridge University Press, Cambridge, 1990, corrected reprint of the 1985 original, https://books.google.com/books? id $=9 \mathrm{wTacO} j \mathrm{HE} 6 \mathrm{IC}$.

[4] O. Karpenkov, Geometry of continued fractions, volume 26 of Algorithms and Computation in Mathematics, Springer, Heidelberg, 2013, doi:10.1007/978-3-642-39368-6.

[5] J. Kuzmanovich and A. Pavlichenkov, Finite groups of matrices whose entries are integers, Amer. Math. Monthly 109 (2002), 173-186, doi:10.2307/2695329.

[6] G. Levitt and V. Metaftsis, Rank of mapping tori and companion matrices, Enseign. Math. (2) 58 (2012), 189-203, doi:10.4171/lem/58-1-9.

[7] G. Mackiw, Finite groups of $2 \times 2$ integer matrices, Math. Mag. 69 (1996), 356-361, doi: $10.2307 / 2691281$.

[8] M. Newman, Integral matrices, Academic Press, New York-London, 1972, pure and Applied Mathematics, Vol. 45, https://www.elsevier.com/books/ integral-matrices/newman/978-0-12-517850-1.

[9] K. i. Tahara, On the finite subgroups of GL(3, Z), Nagoya Math. J. 41 (1971), 169-209, http://projecteuclid.org/euclid.nmj/1118798212.

[10] Q. Yang, Conjugacy classes of torsion in $G L_{n}(\mathbb{Z})$, Electron. J. Linear Algebra 30 (2015), 478-493, doi:10.13001/1081-3810.3110. 